\title{
UNIQUENESS OF SOLUTIONS OF THE DIRICHLET PROBLEM FOR SINGULAR ULTRAHYPERBOLIC EQUATIONS
}

\author{
EUTIQUIO C. YOUNG ${ }^{1}$
}

\begin{abstract}
The paper gives necessary and sufficient conditions for uniqueness of solutions of the Dirichlet problem for ultrahyperbolic partial differential equations with multiple singular lines.
\end{abstract}

1. Introduction. In [1], Diaz and Young obtained necessary and sufficient conditions for the uniqueness of solutions of certain improperly posed problems for the ultrahyperbolic equation

$$
\Delta u-\sum_{j, k=1}^{n}\left(a_{j k} u_{y_{j}}\right)_{y_{k}}+c u=0
$$

where the symbol $\Delta$ denotes the Laplace operator in the variables $x_{1}, \cdots$, $x_{m}$. Their results have been extended recently in [2] to the singular ultrahyperbolic equation

$$
u_{t t}+\frac{\alpha}{t}+\Delta u-\sum_{j, k=1}^{n}\left(a_{j k} u_{y_{j}}\right)_{y_{k}}+c u=0
$$

where $\alpha$ is a real parameter, $-\infty<\alpha<\infty$.

The purpose of this paper is to present some results on the uniqueness of the Dirichlet problem for the class of ultrahyperbolic equations

$$
L u \equiv \sum_{i=1}^{m}\left(u_{x_{i} x_{i}}+\frac{\alpha_{i}}{x_{i}} u_{x_{i}}\right)-\sum_{j, k=1}^{n}\left(a_{j k} u_{y_{j}}\right)_{y_{k}}+c u=0
$$

with multiple singular lines and real parameters $\alpha_{i},-\infty<\alpha_{i}<\infty$.

As usual we consider the boundary value problem in the domain $Q=$ $X \times Y$, where $X$ is a parallelepiped defined by $0<x_{i}<a_{i}(1 \leqq i \leqq m)$ and $Y$

Presented to the Society, January 18, 1972 under the title Uniqueness of the Dirichlet problem for ultrahyperbolic equations with multiple singular lines; received by the editors February 18, 1972.

AMS 1970 subject classifications. Primary 35L10, 35L20; Secondary 35A05.

Key words and phrases. Uniqueness of solution, ultrahyperbolic equations, multiple singular lines, Dirichlet problem, eigenvalues, eigenfunctions, complete set.

${ }^{1}$ Supported by NSF Research Grant GP-11543.

(c) American Mathematical Society 1972 
is a bounded domain in the space $y_{1}, \cdots, y_{n}$. We assume that the coefficients $a_{j k}$ and $c$ are functions of the variables $y_{1}, \cdots, y_{n}$ alone with $c \geqq 0$ in $Y$. Further, we assume that the matrix $\left(a_{j k}\right)$ is symmetric and positive definite, and that $a_{j k}, c$ and the boundary $\partial Y$ of $Y$ are smooth enough to allow the application of the divergence theorem and the existence of a complete set of eigenfunctions for the eigenvalue problem that we will need below.

2. A lemma. An interesting feature of the differential equation (3) is that it possesses several singular lines. As a result of this, every solution of the equation which is smooth on the singular lines satisfies a regularity condition on those lines. This behavior was first observed by Walter [3] in the case of the normal hyperbolic Euler-Poisson-Darboux equation, and later by Fox [4] for a slightly general singular equation. We shall prove this property for our equation (3) as a lemma.

Lemma. If $u \in C^{2}(Q) \cap C^{1}(\bar{Q})$ satisfies $L u=0$, then $u_{x_{i}}=0$ on $x_{i}=0$ for every index $i(1 \leqq i \leqq m)$ for which $\alpha_{i} \neq 0$.

Proof. Let us assume that $\alpha_{m} \neq 0$ and set $x^{\prime}=\left(x_{1}, \cdots, x_{m-1}\right)$ and $z=x_{m}$. Denote by $X^{*}$ the parallelepiped defined by $0<\varepsilon_{i}<x_{i}<a_{i}(1 \leqq i \leqq$ $m-1), 0<z<t\left(t<a_{m}\right)$, and by $X^{\prime}$ the resulting parallelepiped when the variable $z$ is deleted. We integrate over $Q^{*}=X^{*} \times Y$ the identity

$$
\begin{aligned}
2 z^{\beta} u_{z} L u= & {\left[z^{\beta}\left(u_{z}^{2}-u_{x_{i}}^{2}+a_{j k} u_{y j} u_{y_{k}}+c u^{2}\right)\right]_{z} } \\
& +\left(2 z^{\beta} u_{z} u_{x_{i}}\right)_{x_{i}}-\left(2 z^{\beta} a_{j k} u_{z} u_{y_{j}}\right)_{y_{k}} \\
& -\beta z^{\beta-1}\left(u_{z}^{2}-u_{x_{i}}^{2}+a_{j k} u_{y_{j}} u_{y_{k}}+c u^{2}\right) \\
& +2 z^{\beta} u_{z} \alpha_{i} u_{x_{i}} / x_{i}+2 \alpha_{m} z^{\beta-1} u_{z}^{2}
\end{aligned}
$$

where $\beta>0$. Here, as well as in the rest of our discussion, we adopt the convention of summing over repeated indices. Applying the divergence theorem and noting that $u$ is of class $C^{1}$ in $\bar{Q}$, we obtain

$$
\begin{aligned}
& t^{\beta} \int_{Q^{\prime}} F(u) d x^{\prime} d y+\int_{\partial Q^{*}}\left[2 z^{\beta} u_{z}\left(u_{x_{i}} v_{x_{i}}-a_{j k} u_{y_{j}} v_{y_{k}}\right)\right] d S \\
& -\int_{Q^{*}} \beta z^{\beta-1}\left[F(u)-2 z u_{z} \alpha_{i} u_{x_{i}} /\left(\beta x_{i}\right)-2 \alpha_{m} u_{z}^{2} / \beta\right] d x^{\prime} d y d z=0
\end{aligned}
$$

where

$$
F(u)=u_{z}^{2}-u_{x i}^{2}+a_{j k} u_{y_{j}} u_{y k}+c u^{2},
$$

$Q^{\prime}=X^{\prime} \times Y$ and $\left(v_{x_{i}}, v_{y_{k}}\right)$ is the outward unit normal vector on $\partial Q^{\prime}$.

Let us divide (4) throughout by $t^{\beta}$ and take the limit as $t$ approaches zero. We observe that the second integral drops out in the limit as its integrand is bounded and $(z \mid t)<1$. On the other hand, the third integral becomes a 
derivative with respect to $z^{\beta}$ at $z=0$ as may be seen by writing it in the form

$$
\frac{1}{t^{\beta}} \int_{0}^{t^{\beta}} \int_{Q^{\prime}}\left[F(u)-2 z u_{z} \alpha_{i} u_{x_{i}} /\left(\beta x_{i}\right)-2 \alpha_{m} u_{z}^{2} / \beta\right] d x^{\prime} d y d\left(z^{\beta}\right) .
$$

Thus, in the limit, we obtain from (4)

$$
\left(2 \alpha_{m} / \beta\right) \int_{Q^{\prime}} u_{z}^{2}\left(x^{\prime}, 0, y\right) d x^{\prime} d y=0 .
$$

Since $\alpha_{m} \neq 0$, this implies that $u_{z}\left(x^{\prime}, 0, y\right)=0$ in $Q^{\prime}$. By continuity, this holds for $0 \leqq x_{i} \leqq a_{i}(1 \leqq i \leqq m-1)$ and for all $y$ in $\bar{Y}$.

3. The Dirichlet problem. Let us now consider the homogeneous Dirichlet problem

$$
L u=0 \quad \text { in } Q, \quad u=0 \quad \text { on } \partial Q .
$$

By changing subscripts if necessary, we can assume that $\alpha_{i}, i=1, \cdots, p$, are not necessarily zero and $\alpha_{j}=0, j=p+1, \cdots, m$ with $0 \leqq p \leqq m$.

THEOREM 1. If $\alpha_{i}>0$ for all $i(1 \leqq i \leqq p)$, then every solution $u \in C^{2}(Q) \cap$ $C^{1}(\bar{Q})$ of the problem (5) vanishes identically in $Q$.

Proof. Suppose that $u \in C^{2}(Q) \cap C^{1}(\bar{Q})$ is a solution of (5). By the hypothesis, it follows from the lemma that $u_{x_{i}}=0$ on $x_{i}=0$ for $i=1, \cdots, p$. Now let us integrate over $Q$ the identity

$$
\begin{aligned}
2 u_{x_{r}} L u \equiv & \left(2 u_{x_{r}} u_{x_{i}}\right)_{x_{i}}-\left(u_{x_{i}}^{2}-a_{j k} u_{y_{j}} u_{y_{k}}-c u^{2}\right)_{x_{r}} \\
& -\left(2 a_{j k} u_{x_{r}} u_{y_{j}}\right)_{y_{k}}+2 u_{x_{r}} \alpha_{i} u_{x_{i}} / x_{i} \\
= & 0
\end{aligned}
$$

$(1 \leqq r \leqq p)$ and use the divergence theorem. Because $u=0$ on $\partial Q$ and $u_{x_{i}}=0$ on $x_{i}=0$ for $i=1, \cdots, p$, we obtain

$$
\int_{X_{r} \times Y^{r}} u_{x_{r}}^{2}\left(a_{r}, x^{\prime}, y\right) d x^{\prime} d y+2 \int_{Q} u_{x_{r}} \alpha_{i} u_{x_{i}} / x_{i} d x d y=0
$$

where $X_{r}$ denotes the parallelepiped defined by $0<x_{i}<a_{i}, 1 \leqq i \leqq m, i \neq r$, and $x^{\prime}$ denotes a point in $X_{r}$. Taking the sum of (6) with respect to $r$ leads then to the equation

(7) $\sum_{r=1}^{p} \int_{X_{r} \times Y} u_{x_{r}}^{2}\left(a_{r}, x^{\prime}, y\right) d x^{\prime} d y+2 \int_{Q}\left(\sum_{r=1}^{n} u_{x_{r}}\right)\left(\sum_{i=1}^{n} \alpha_{i} u_{x_{i}} / x_{i}\right) d x d y=0$ where we observe that each of the summands in the first sum is nonnegative. 
Now, by the Cauchy-Schwarz inequality, we have

$$
\left(\sum_{i=1}^{p} u_{x_{i}}\right)\left(\sum_{i=1}^{p} \alpha_{i} u_{x_{i}} / x_{i}\right) \geqq\left(\sum_{i=1}^{p}\left(\alpha_{i} / x_{i}\right)^{1 / 2} u_{x_{i}}\right)^{2} \geqq 0 .
$$

Therefore, the integrand of the second integral in (7) is also nonnegative. Hence we conclude, from (7) and (8), that

$$
\sum_{i=1}^{n}\left(\alpha_{i} / x_{i}\right)^{1 / 2} u_{x_{i}}=0 .
$$

If we multiply (9) by $2 u$ and integrate the result over the domain $D$ defined by $0<x_{i}<a_{i}(1 \leqq i \leqq p)$, we finally obtain

$$
\sum_{i=1}^{p} \int_{D}\left(\alpha_{i}^{1 / 2} u^{2} / x_{i}^{3 / 2}\right) d x_{1} \cdots d x_{p}=0
$$

since $u=0$ on $\partial D$. This yields the conclusion of the theorem.

When the parameters $\alpha_{i}$ are all nonpositive, we have the following result.

THEOREM 2. Let $\lambda_{k}(k=1,2, \cdots)$ be the eigenvalues of the problem

$$
\begin{aligned}
\left(a_{j k} v_{y_{j}}\right)_{y_{k}}-c v+\lambda v & =0 & & \text { in } Y, \\
v & =0 & & \text { on } \partial Y .
\end{aligned}
$$

If $\alpha_{i} \leqq 0$ for $i=1, \cdots, p$, then every solution $u \in C^{2}(Q) \cap C^{1}(\bar{Q})$ of the problem (5) vanishes identically in $Q$ if, and only if, for all nonzero real numbers $\mu_{1}, \cdots, \mu_{n}$ and for all nonzero integers $b_{p+1}, \cdots, b_{m}$ satisfying the condition

$$
\sum_{i=1}^{p} \mu_{i}+\sum_{i=p+1}^{m}\left(b_{i} \pi x_{i} / a_{i}\right)^{2}=\lambda_{k}
$$

there exists $\mu_{r}(1 \leqq r \leqq p)$ such that

$$
J_{\left(1-\alpha_{r}\right) / 2}\left(\mu_{r}^{1 / 2} a_{r}\right) \neq 0
$$

where $J_{p}(t)$ denotes the Bessel function of the first kind of order $p$.

ProOf. The necessity part follows easily. Let $\lambda_{s}$ be an eigenvalue of (10) and $v_{s}$ a corresponding eigenfunction. Suppose that there exist nonzero real numbers $v_{1}, \cdots, v_{p}$ and nonzero integers $q_{p+1}, \cdots, q_{m}$ satisfying (11) such that

$$
J_{\left(1-\alpha_{i}\right) / 2}\left(\nu_{i}^{1 / 2} a_{i}\right)=0, \quad i=1, \cdots, p .
$$

Then the function

$$
u(x, y)=\prod_{i=1}^{n} x_{i}^{\left(1-\alpha_{i}\right) / 2} J_{\left(1-\alpha_{i}\right) / 2}\left(v_{i}^{1 / 2} x_{i}\right) \phi(x ; q) v_{s}(y)
$$


where

$$
\phi(x ; q)=\prod_{i=p+1}^{m} \sin \left(q_{i} \pi x_{i} / a_{i}\right)
$$

is a nontrivial solution of the problem (5) as is readily verified.

Conversely, suppose that condition (12) holds. Let $\lambda_{k}$ be an eigenvalue of (10) and let $\mu_{i}$ be any nonzero real numbers such that

$$
J_{\left(1-\alpha_{i}\right) / 2}\left(\mu_{i}^{1 / 2} a_{i}\right)=0
$$

for all $i(1 \leqq i \leqq p)$ except for $i=r$. For any nonzero integers $b_{p+1}, \cdots, b_{m}$, let $\mu_{r}$ be determined by the relation (11). Notice that $\mu_{r}$ may very well be a negative number and that from the hypothesis we have

$$
J_{\left(1-x_{r}\right) / 2}\left(\mu_{r}^{1 / 2} a_{r}\right) \neq 0 .
$$

Now let

$$
w(x, y)=\prod_{i=1}^{p} x_{i}^{\left(1+\alpha_{i}\right) / 2} J_{\left(1-\alpha_{i}\right) / 2}\left(\mu_{i}^{1 / 2} x_{i}\right) \phi(x ; b) v_{k}(y)
$$

where $\phi(x ; b)$ is defined in (13) and $v_{k}$ is an eigenfunction of (10) corresponding to $\lambda_{k}$. By direct differentiation, making use of (10) and (11), it is readily shown that (16) satisfies the adjoint equation

$$
M w \equiv w_{x_{i} x_{i}}-\alpha_{i}\left(w / x_{i}\right)_{x_{i}}-\left(a_{j k} w_{y_{j}}\right)_{y_{k}}+c w=0
$$

of (3). Moreover, from (13), (14), and (15), it is clear that (16) vanishes on $\partial Q$ except on $x_{r}=a_{r}$.

Consider the Green's formula

$$
\begin{aligned}
\int_{Q s}(w L u-u M w) d x d y \\
\quad=\int_{\partial Q s}\left[\left(w u_{x_{i}}-u w_{x_{i}}+\left(\alpha_{i} / x_{i}\right) u w\right) v_{x_{i}}-a_{j k}\left(w u_{y_{j}}-u w_{y_{j}}\right) v_{y_{k}}\right] d S
\end{aligned}
$$

where $Q_{s}=X_{s} \times Y, X_{s}$ being the parallelepiped defined by $0<s_{i}<x_{i}<a_{i}$ for $i=1, \cdots, p$, and $0<x_{i}<a_{i}$ for $i=p+1, \cdots, m$, and $\left(v_{x_{i}}, v_{y_{k}}\right)$ is the outward unit normal vector on $\partial Q_{s}$. If $u$ is a solution of (5) and $w$ is chosen as the function (16), then substitution of these functions in (17) yields

$$
\int_{\partial Q_{s}}\left(w u_{x_{i}}-u w_{x_{i}}+\left(\alpha_{i} / x_{i}\right) u w\right) v_{x_{i}} d S=0 .
$$

Now let $s_{i}(1 \leqq i \leqq p)$ approach zero. Since $w_{x_{i}}$ and $w / x_{i}$ are bounded at $x_{i}=0$ for $i=1, \cdots, p$, and $u=0$ on $x_{i}=0, x_{i}=a_{i}$ for all $i, 1 \leqq i \leqq m$, it 
follows from (13), (14), and (16) that (18) reduces to

$$
a_{r}^{\left(1+\alpha_{r}\right) / 2} J_{\left(1-\alpha_{r}\right) / 2}\left(\mu_{r}^{1 / 2} a_{r}\right) \cdot \int u_{x_{r}}\left(a_{r}, x^{\prime}, y\right) \psi(x ; \mu) \phi(x ; b) v_{k}(y) d x^{\prime} d y=0
$$

where

$$
\psi(x ; \mu)=\prod_{i=1 ; i \neq r}^{p} x_{i}^{\left(1+\alpha_{i}\right) / 2} J_{\left(1-\alpha_{i}\right) / 2}\left(\mu_{i}^{1 / 2} x_{i}\right) .
$$

$X^{\prime}$ is the subspace of $X$ with $x_{r}$ deleted and $x^{\prime}$ denotes a point in $X^{\prime}$. In view of (15), we conclude that

$$
\int_{X^{\prime} \times Y} u_{x_{r}}\left(a_{r}, x^{\prime}, y\right) \psi(x ; \mu) \phi(x ; b) v_{k}(y) d x^{\prime} d y=0
$$

for all nonzero $\mu_{i}$ numbers satisfying (14), for all nonzero integers $b_{p+1}$, $\cdots, b_{m}$, and for all eigenfunctions $v_{k}$. By the completeness of the sets of eigenfunctions $\{\psi(x ; \mu)\},\{\phi(x ; b)\}$, and $\left\{v_{k}\right\}$ in the respective spaces $\left\{x_{i} \mid 0<x_{i}<a_{i}, 1 \leqq i \leqq p, i \neq r\right\},\left\{x_{i} \mid 0<x_{i}<a_{i}, p+1 \leqq i \leqq m\right\}$, and $Y$, we conclude that

$$
u_{x_{r}}=0 \text { on } x_{r}=a_{r} .
$$

Finally, by integrating the identity

$$
\begin{aligned}
\left(2 x_{r} u_{x_{r}}+u\right) L u= & {\left[x_{r}\left(-u_{x_{i}}^{2}+a_{j k} u_{y_{j}} u_{y_{k}}+c u^{2}\right)\right]_{x_{r}} } \\
& +\left[2 x_{r} u_{x_{r}} u_{x_{i}}+u u_{x_{i}}+\left(\alpha_{i} u^{2}\right) /\left(2 x_{i}\right)\right]_{x_{i}} \\
& -\left[a_{j k}\left(2 x_{r} u_{x_{r}}+u\right) u_{y_{j}}\right]_{y_{k}} \\
& -\left(2 u_{x_{r}}^{2}-\left(\alpha_{i} u^{2}\right) /\left(2 x_{i}^{2}\right)-2 x_{r} u_{x_{r}}\left(\alpha_{i} u_{x_{i}} / x_{i}\right)\right.
\end{aligned}
$$

over $Q$ and using (21), we obtain

$$
\int_{Q}\left(2 u_{x_{r}}^{2}-\frac{u^{2} \alpha_{i}}{2 x_{i}^{2}}-2 x_{r} u_{x_{r}} \frac{\alpha_{i}}{x_{i}} u_{x_{i}}\right) d x d y=0 .
$$

Since $r$ is arbitrary, summing (22) with respect to $r$ from 1 to $p$ yields

$$
\int_{Q}\left[2 \sum u_{x_{i}}^{2}-\frac{p u^{2}}{2} \sum\left(\alpha_{i} / x_{i}^{2}\right)-2 \sum\left(x_{i} u_{x_{i}}\right) \sum\left(\alpha_{i} u_{x_{i}} / x_{i}\right)\right] d x d y=0
$$

where we have dropped the limits of summation for convenience. Since $\alpha_{i} \leqq 0,1 \leqq i \leqq p$, we see by the Cauchy-Schwarz inequality that

$$
\left(\sum\left(-\alpha_{i}\right)^{1 / 2} u_{x_{i}}\right)^{2} \leqq-\sum\left(x_{i} u_{x_{i}}\right) \sum\left(\alpha_{i} u_{x_{i}} / x_{i}\right)
$$

so that $\sum\left(x_{i} u_{x_{i}}\right) \sum\left(\alpha_{i} u_{x_{i}} / x_{i}\right) \leqq 0$. It follows that the integrand in (23) is 
nonnegative from which we conclude that $u$ vanishes identically in $Q$. This completes the proof of the theorem.

\section{REFERENCES}

1. J. B. Diaz and E. C. Young, Uniqueness of solutions of certain boundary value problems for ultrahyperbolic equations, Proc. Amer. Math. Soc. 29 (1971), 569-574.

2. E. C. Young, Uniqueness of solutions of improperly posed problems for singular ultrahyperbolic equations, J. Austral. Math. Soc. (to appear).

3. W. Walter, Über die Euler-Poisson-Darboux-Gleichung, Math. Z. 67 (1957), 361376. MR 19, 1059.

4. D. W. Fox, The solution and Huygens' principle for a singular Cauchy problem, J. Math. Mech. 8 (1959), 197-219. MR 21 \#762.

Department of Mathematics, Florida State University, Tallahassee, Florida 32306 